\title{
THE ROLE OF SPECIES ABUNDANCE IN DETERMINING BREEDING ORIGINS OF MIGRATORY BIRDS WITH STABLE ISOTOPES
}

\author{
J. Andrew Royle ${ }^{1,3}$ And Dustin R. Rubenstein ${ }^{2}$ \\ ${ }^{1}$ U.S. Fish and Wildlife Service, Division of Migratory Bird Management, 11510 American Holly Drive, \\ Laurel, Maryland 20708 USA \\ ${ }^{2}$ Cornell University, Department of Neurobiology and Behavior, Seeley G. Mudd Hall, Ithaca, New York 14853-2702 USA
}

\begin{abstract}
Understanding the breeding origins of migratory birds captured on their wintering grounds has important management and conservation implications for declining populations of songbirds. Stable isotopes have recently been used to infer origins for species where application of conventional markers fails. A natural method of linking wintering birds to breeding populations, and one that has not been previously applied to stable isotopes, is based on likelihood. Using a likelihood assignment rule, birds are associated with the breeding population under which their realized isotope signature is most likely to have been generated. We report the first illustration of using likelihood-based assignment with stable isotope data. Moreover, within a probability-based framework for assignment, we argue that a more natural formulation of the assignment problem should be based on the probability of origin given the observed data, or the posterior probability of origin. The relationship between posterior assignment and that based on simple likelihood is embodied in Bayes Rule, which establishes a clear linkage between the distribution of the breeding population (i.e., relative abundance) and probability of origin. We demonstrate likelihood and posterior assignment using a large data set on Black-throated Blue Warblers (Dendroica caerulescens). Our results suggest that relative abundance is likely to be a more crucial consideration in the presence of less geographically structured isotopes, when the distribution of abundance is highly non-uniform, or when the range of the species is geographically restricted.

Key words: Bayes Rule; Black-throated Blue Warbler; breeding origins; connectivity; Dendroica caerulescens; likelihood assignment; migratory birds; relative abundance; species abundance; stable isotopes.
\end{abstract}

\section{INTRODUCTION}

Establishing the movement patterns of migratory bird populations is essential for the conservation and management of declining avian species. Moreover, determining the degree of migratory connectivity, or the links between breeding and nonbreeding populations (Webster et al. 2002), is important for understanding a species' ecology and life history. There are several techniques for investigating connectivity and movement in migratory populations. The most common approaches use extrinsic markers (e.g., bird rings, radio telemetry) or exploit natural, intrinsic biological (e.g., behavioral, morphological, or genetic variation) or biogeochemical (e.g., trace element concentrations, stable isotope signatures) markers (Webster et al. 2002, Rubenstein and Hobson 2004). Extrinsic markers rely mostly on direct observation or recovery of banded individuals, while intrinsic markers exploit natural variation (e.g., in stable isotope signatures or genetic variation) among populations (Rubenstein and Hobson 2004). A shared objective of these diverse tools is to

Manuscript received 21 January 2004; accepted 5 March 2004; final version received 22 April 2004. Corresponding Editor (ad hoc): J. J. Pastor.

${ }^{3}$ E-mail: andy_royle@fws.gov infer the breeding origin of birds captured at some location on their wintering grounds, hereafter known as "the assignment problem." The assignment problem has a long history in waterfowl management, where it is known as "derivation of harvest" (Nichols and Tomlinson 1993), and estimates of the contribution of different (geographic) breeding populations to the harvest are based on band recoveries. Similarly, in fisheries management, the analogous problem of identifying the contribution of various populations to catch, known as "genetic stock identification" or "mixed stock analysis," has been based on conventional tags and genetic markers (Utter and Ryman 1993). Traditional approaches using banding data of migratory passerine birds to examine migratory connectivity have proved impractical because, even though considerable numbers of birds may have been banded, there have been few recoveries for most species (Hobson 2004, in press). Stable isotopes offer a promising new method for unraveling avain movement patterns, but rigorous analytical and statistical approaches to analyze isotope data are still lacking (Hobson 2004, in press).

With stable isotope markers, the isotopic signature of a tissue reflects the isotopic signature of the local environment where the tissue was grown (Chamberlain et al. 1997, Hobson and Wassenaar 1997). Consequent- 
ly, when variation in tissue isotopic signatures across the breeding range (for tissues that reflect breeding isotope signatures) is quantified, some ability to infer breeding origin of wintering birds is possible (Chamberlain et al. 1997, Hobson and Wassenaar 1997, 2001, Hobson et al. 2001, Kelly et al. 2002, Rubenstein et al. 2002). However, assignment of an individual caught on the wintering grounds to a specific breeding area is imperfect because there may be considerable variation in isotopic structure, even among birds sampled at the same breeding location (e.g., Graves et al. 2002), or because there are large geographic areas with similar isotopic baselines (e.g., latitudinal bands of $\delta D$ ). This is in contrast to the use of banding information for studying movement, because banding yields a precise observation of origin (i.e., location of banding). Unlike banding data, however, stable isotope markers do not rely on recapturing specific individuals, which makes them potentially more powerful than traditional extrinsic markers for small, long-distance migrants that can be nearly impossible to recapture or resight (Rubenstein and Hobson 2004).

Overcoming the difficulties in assignment, given the imprecise linkage between isotope signature and breeding origin, will be essential for making stable isotopes a powerful tool in unraveling animal movement patterns. Such difficulties are also an element of studies that employ genetic markers, and assignment based on likelihood is commonly used to address this issue (see sidebar in Webster et al. 2002). That is, an individual whose origin or population is unknown is assigned to that population under which the realized value of its marker is most likely to have originated. Geographic variation in abundance is an important consideration in developing assignment rules, regardless of what sort of marking technique is used. Moreover, the role of abundance in determining origin has been considered when inference is based on either genetic marker or banding data, but its role has not been recognized in studies involving isotope markers. In this paper, we (1) consider likelihood-based methods of determining breeding origins using stable isotope markers and (2) establish the role that geographic variation in species abundance plays in the problem. This is the first study to use isotope markers to infer migratory connectivity with assignments based on likelihood and while accounting for geographic variation in species abundance.

\section{The Stable Isotope Assignment Problem}

Stable isotopes are naturally occurring elemental forms with different nuclear masses that are measured as isotopic differences relative to a known standard. Isotope ratios are often expressed as ratios in units of parts per thousand (\%o) (or "per mil") according to the following equation:

$$
\delta X_{\text {std }}=\left(R_{\text {sample }} / R_{\text {std }}-1\right) \times 1000
$$

where $\delta X_{\text {std }}$ is the isotope ratio in delta units relative to a standard and $R_{\text {sample }}$ and $R_{\text {std }}$ are the absolute isotope ratios of the sample and standard, respectively. The arbitrary choice of standards, e.g., the PeeDee belemnite (PDB) standard for carbon and the standard mean ocean water (SMOW) for hydrogen, results in many isotope values (e.g., both carbon and hydrogen) typically being negative.

Stable isotope signatures in animal tissues reflect those of local food webs (e.g., Peterson and Fry 1987). Isotopes are incorporated directly from the diet into animal tissues with varying degrees of trophic enrichment, or the difference in isotope ratios between an animal and its putative diet. For some isotopes, such as hydrogen and carbon, tissue isotope ratios accurately reflect those of their diet with little enrichment, whereas others, such as nitrogen, show considerable enrichment and are affected by water and nutritional stress (reviewed in Rubenstein and Hobson 2004). This makes carbon and hydrogen isotopes particularly useful for studying animal movements.

The rationale underlying the use of isotopes for determining origin is that certain isotopes exhibit distinct natural, geographic patterns of variation (due to a variety of biogeochemical and biological processes; reviewed in Rubenstein and Hobson 2004), which are reflected in tissues (Chamberlain et al. 1997, Hobson and Wassenaar 1997), in this case, feathers grown in the breeding area. Thus, observation of the isotope signature in a bird of unknown origin can be informative about its breeding origin, or location at the time of molt. The nominal goal then, is to infer the origin of a bird caught at some location within its wintering range, given information about the isotopic structure contained in the bird's feathers.

For clarity, assume that animals originate from $B$ discrete breeding populations, say $b=1,2, \ldots, B$ and migrate to $w=1,2,3, \ldots, W$ wintering locations. The term population is used loosely here in a metapopulation sense, wherein the population might actually refer to geographically indexed collections of individuals or simply to geographic strata. Measuring a single isotope, $y\left(y \equiv \delta X_{\text {std }}\right.$ in Eq. 1), for a large number of birds from some breeding population (i.e., within some geographic stratum) yields an estimate of the probability distribution of $y$ for that stratum: the distribution of $y$ conditional on $b, f(y \mid b)$. Hereafter, various probability distribution functions will be indicated by $f(u)$ (for the marginal probability distribution function of $u$ ) or $f(u \mid(v)$ (for the conditional probability distribution function of $u$ given $v$ ). Ideally we would characterize $f(y \mid b)$ for continuous $b$. However, because the number of populations sampled is finite, and because a population usually contains a trivial fraction of the species, it is necessary to assume that sampled populations are representative of broader areas in order for an estimate of $f(y \mid b)$ to have any relevance. 
Sampling $y$ in $B$ strata throughout the breeding range yields a set of estimated probability distributions $f(y \mid b) ; b=1,2, \ldots . B$. Sampling a feather from a random bird in a wintering population yields a measurement, $y^{*}$, of the isotope concentration in that feather. An intuitive approach for associating the bird that generated $y^{*}$ to some $b$ is to assign $y^{*}$ to that breeding origin for which its isotopic signature is most likely to have been generated. To determine the most likely origin, the likelihood can be evaluated for each $b, f(y \mid b)$, fixing $y=y^{*}$. The likely origin of the bird generating $y^{*}$ is that $b$ which has the largest $f\left(y^{*} \mid b\right)$.

For some problems, it may be reasonable to assume that $f(y \mid b)$ is normal, with mean, ( $\mu_{b}$, and standard deviation, $\sigma_{b}$, estimated from sample data. Then, the likelihood corresponding to an observation $y^{*}$ is

$$
f\left(y^{*} \mid \mu_{b}, \sigma_{b}\right)=\frac{1}{\sqrt{2 \pi} \sigma_{b}} \exp \left[-\frac{1}{2 \sigma_{b}^{2}}\left(y^{*}-\mu_{b}\right)^{2}\right]
$$

which may be computed for each $\left(\mu_{b}, \sigma_{b}\right)$.

\section{Illustration}

Suppose we have characterized the isotopic structure over the breeding range of some avian species over two geographic regions, or populations. Based on a large number of samples, we conclude that the first population has mean $\mu_{1}=-144 \%$ and a standard deviation $\sigma_{1}=4 \%$, and the second population has mean $\mu_{2}=$ $-152 \%$ o with standard deviation $\sigma_{2}=3 \%$ (subsequently, we suppress indication of the units unless needed to avoid confusion).

Now, given an observation $y^{*}$ collected from a bird captured at some wintering location, the goal is to determine the most likely population of origin for $y^{*}$. Suppose we observe $y^{*}=-151$, then the competing likelihoods are $f\left(y=-151 \mid \mu_{1}, \sigma_{1}\right)=0.02157$ and $f(y$ $\left.=-151 \mid \mu_{2}, \sigma_{2}\right)=0.12579$. Consequently, the bird that produced $y^{*}=-151$ appears much more likely to have originated from population 2 than 1 . Supposing that $y^{*}=-148$ (precisely between $\mu_{1}$ and $\mu_{2}$ ), then $f\left(y^{*} \mid b=1\right)=0.0605$ and $f\left(y^{*} \mid b=2\right)=0.0547$. We see that population 1 is only slightly more likely to have generated the observation owing to its larger variance, which results in more mass in the vicinity of -148 . It may be useful to characterize assignment in terms of a "probability of origin" interpretation, normalizing the likelihood according to

$$
\pi_{b}=\frac{f\left(y=y^{*} \mid b\right)}{\sum_{b=1}^{B} f\left(y=y^{*} \mid b\right)} .
$$

Normalizing these values yields the probabilities 0.525 (of originating from $b=1$ ), and 0.475 (of originating from $b=2$ ) for the observation $y^{*}=-148$. It is tempting to interpret $\pi_{b}$ as the "probability of origin $b$ given the observation $y^{*}$ ", i.e., $f\left(b \mid y=y^{*}\right)$. Although $\pi_{b}$ may not be an unreasonable assessment of the condi-

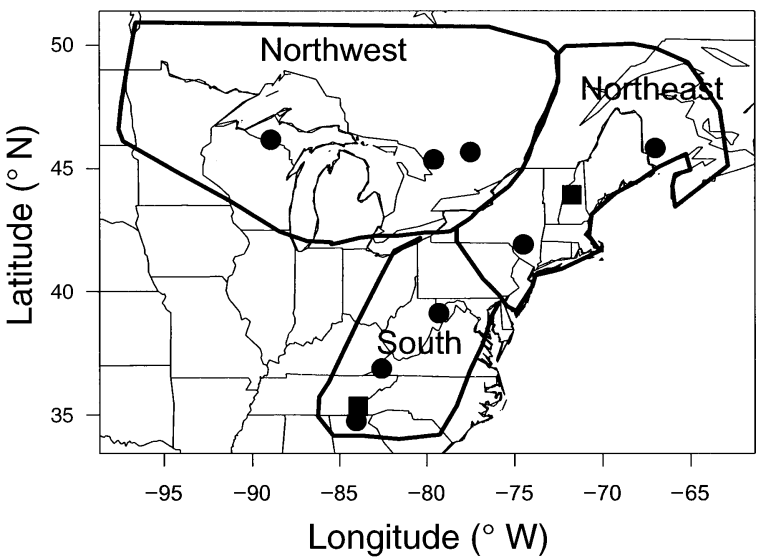

Fig. 1. Geographic strata and sample locations used in analysis of the Black-throated Blue Warbler isotopic data. Solid circles indicate data on $\delta^{13} C$ and $\delta D$ isotopes; solid squares indicate data on $\delta^{13} C$ only.

tional probability $f\left(b \mid y=y^{*}\right)$, one should consider this interpretation cautiously. This is discussed further in The Importance of Relative Abundance.

It is easy to extend this notion of likelihood-based assignment to multiple isotope systems, in which case $f(y \mid b)$ might be a multivariate normal likelihood with variance-covariance matrix $\Sigma_{p \times p}$ for a $p$-isotope system. This is considered in the following section.

\section{AssignMENT AND BLACK-THROATED Blue WARBLERS}

Here we use data from the Black-throated Blue Warbler (Dendroica caerulescens). Carbon $\left(\delta^{13} C\right)$ and hydrogen $(\delta D)$ isotopes were analyzed in feathers from Black-throated Blue Warblers collected at 10 sites throughout their temperate North American breeding range (Fig. 1; for a description of the analytical methods, see Rubenstein et al. 2002). Both $\delta^{13} C$ and $\delta D$ were measured on 129 individuals from eight of these sites, and $\delta^{13} C$ was measured on an additional 137 individuals from all 10 sites (see Rubenstein et al. 2002).

Carbon and hydrogen isotopes in feathers of Blackthroated Blue Warblers show a strong latitudinal pattern, tending to decrease with breeding latitude (Chamberlain et al. 1997, Rubenstein et al. 2002). We stratified the Black-throated Blue Warbler breeding range into three geographic strata corresponding roughly to the northwestern (NW), northeastern (NE), and southern (S) portions of the Black-throated Blue Warbler range (Fig. 1). Both isotopes showed significant variation among these three regions (for carbon, $F_{2,263}=$ 41.99, $P<0.001$; for hydrogen, $F_{2,126}=50.99, P<$ 0.001; Table 1).

We consider likelihood-based assignment assuming normal distributions for $f(y \mid b)$, with the parameters estimated from the sample data (Table 1). To evaluate performance, we consider the assignment outcomes using the 266 observations compared with that based on 
TABLE 1. Stable isotope means and standard deviations (\%o) from feathers of Black-throated Blue Warblers for each North American breeding region.

\begin{tabular}{lcclcc}
\hline \hline \multirow{2}{*}{ Region } & \multicolumn{2}{c}{$\delta^{13} C$} & & \multicolumn{2}{c}{$\delta D$} \\
\cline { 2 - 3 } \cline { 5 - 6 } \cline { 5 - 6 } & $\mu$ & $\sigma$ & & $\mu$ & $\sigma$ \\
\hline Northwest & -24.18 & 0.72 & & -96.84 & 11.95 \\
Northeast & -24.50 & 0.85 & & -74.88 & 11.33 \\
South & -23.34 & 1.03 & & -75.52 & 12.38 \\
\hline
\end{tabular}

Note: Data are from Rubenstein et al. (2002).

a random assignment rule under which observations are assigned to each region with probability $1 / 3$. Using this null assignment rule, we would expect to obtain a correct classification rate of $\sim 33 \%$. This is obtained by nothing that the fraction of the $\delta^{13} C$ and $\delta D$ observations from each region were $(0.323,0.327,0.350)$ and $(0.388,0.264,0.349)$, respectively. Thus, the probability that an observation is assigned to the correct region is the sum of these fractions multiplied by $1 / 3$ (in both cases, this is $\sim 33 \%$ ).

Using each isotope alone, 135 out of 266 correct assignments (50.8\%) were generated based on $\delta^{13} \mathrm{C}$ and 69 out of 129 correct $(53.5 \%)$ assignments were made based on $\delta D$. Thus, each isotope yields an improvement in correct assignment rate with respect to random assignment $(33 \%)$. In an attempt to improve on this, we considered assignment based jointly on the pair of isotopes. For this, the multivariate $f(y \mid b)$ is characterized by an additional parameter, the covariance between the pair of isotopes under consideration. The bivariate normal $f(y \mid b)$ depends on the $2 \times 2$ variance covariance matrix:

$$
\sum=\left[\begin{array}{cc}
\sigma^{2}\left(\delta^{13} C\right) & \sigma\left(\delta^{13} C, \delta D\right) \\
\sigma\left(\delta D, \delta^{13} C\right) & \sigma^{2}(\delta D)
\end{array}\right] .
$$

For purposes here, $\sigma\left(\delta^{13} C, \delta D\right)$ was assumed to be constant among regions and thus was estimated from all 266 observations. Use of both $\delta^{13} C$ and $\delta D$ yields 156 correct $(58.7 \%)$ assignments, a slight improvement over that based on use of $\delta D$ alone. Recall that $137 \delta D$ observations were missing and thus assignment for these observations was based on carbon alone. Use of only that subset of 129 observations for which both isotopes were measured yields 80 correct assignments $(62 \%)$.

\section{Wintering birds}

Here, we consider the isotope data from 384 birds sampled on four Caribbean islands (Cuba, Jamaica, Hispaniola, and Dominican Republic) that span most of the Black-throated Blue Warbler's wintering range in the Greater Antilles (see Rubenstein et al. 2002). To determine the breeding origin of these 384 individuals, we considered single-isotope assignment based on $\delta^{13} C$, and using both $\delta^{13} C$ and $\delta D$ for those samples where $\delta D$ data were available (144 individuals). The origin frequencies for birds from each of the four is- lands are given in Table 2. We note that there is a considerable difference between the origin distributions, depending on whether only $\delta^{13} C$, or both isotopes, are used. The fact that $\delta D$ is the more geographically differentiated of the two would favor the latter result. Rubenstein et al. (2002) identified a northern origin for birds found wintering in Cuba and Jamaica and a southern origin for birds found wintering in the Dominican Republic and Puerto Rico. The results given in Table 2 broadly agree with those reported by Rubenstein et al. (2002); the indicated distributions of birds from Cuba and Jamaica based on both isotopes were (NW, NE, S): $(49 \%, 49 \%, 2 \%)$ and $(58 \%, 27 \%, 15 \%)$, respectively, whereas for the Dominican Republic and Puerto Rico the distributions were $(32 \%, 11 \%, 57 \%)$ and $(20 \%, 30 \%, 50 \%)$, respectively.

\section{The Importance of Relative Abundance}

As we have demonstrated, the determination of breeding origin may be carried out without regard to the spatial distribution of abundance of that species being considered. However, variation in abundance may be an important consideration in many problems. To motivate this, consider two distinct populations consisting of 1000 and 100 individuals, and assume that there exists a discrete marker (e.g., an allele) taking on values 1, 2, and 3. Suppose that the frequencies of individuals in each population having each value of $y$ are according to Table 3 . The probability distribution of $y$ for each population is also indicated.

Now suppose that the value $y^{*}=3$ is observed from some bird drawn randomly from the population. We see that the likelihood $f(y=3 \mid b=2)$ is six times that of $f(y=3 \mid b=1)$, which would appear to support the belief that population 2 is the likely origin of $y^{*}$. However, note that there are $60 \%$ more birds in population 1 , which possess $y=3$ (i.e., 50 out of 80 with $y=3$ in the population at large). Thus, a bird with $y=3$ chosen at random from the population is, in fact, $60 \%$ more likely to have originated from $b=1$ than $b=2$ despite the contradictory likelihood of such an obser-

TABLE 2. Frequency of origin for birds from four Caribbean islands for likelihood-based assignment based on carbon isotopic data alone and using both carbon and hydrogen isotopes.

\begin{tabular}{lcccc}
\hline \hline & \multicolumn{4}{c}{ Wintering capture location } \\
\cline { 2 - 5 } $\begin{array}{l}\text { Predicted } \\
\text { origin }\end{array}$ & Cuba & $\begin{array}{c}\text { Dominican } \\
\text { Republic }\end{array}$ & $\begin{array}{c}\text { Puerto } \\
\text { Rico }\end{array}$ & Jamaica \\
\hline$\delta^{13} C$ & & & & \\
Northwest & 31 & 15 & 20 & 95 \\
Northeast & 54 & 6 & 7 & 61 \\
South & 3 & 35 & 17 & 39 \\
$\delta^{13}$ C and $\delta D$ & & & & \\
Northwest & 43 & 18 & 9 & 113 \\
Northeast & 43 & 6 & 13 & 52 \\
South & 2 & 32 & 22 & 30 \\
\hline
\end{tabular}

Note: Data are from Rubenstein et al. (2002). 
TABLE 3. Data illustrating the potential effect of variation in abundance of two populations on assignment. Rows represent a discrete marker taking on three values $(1,2,3)$.

\begin{tabular}{ccc}
\hline \hline $\begin{array}{l}\text { Population and } \\
\text { marker value }\end{array}$ & Frequency & $f(y \mid b) \dagger$ \\
\hline$N_{1}=1000$ & & \\
1 & 800 & 0.80 \\
2 & 150 & 0.15 \\
3 & 50 & 0.05 \\
$N_{2}=100$ & & \\
1 & 20 & 0.20 \\
2 & 50 & 0.50 \\
3 & 30 & 0.30 \\
\hline
\end{tabular}

$\dagger$ For $N_{1}, b=1$; for $N_{2}, b=2$.

vation. This apparently contradictory inference about origin is a consequence of variable abundance that has not been considered in assignment based simply on the likelihood.

To clarify this, it is necessary to introduce an alternative quantity upon which to base assignment. In probability terms, the assignment problem is naturally stated in terms of the conditional probability $f(b \mid y)$, the probability of $b$ given $y$ (observed at some wintering locale). Note that sampling of birds within their breeding range generates data that lead to a characterization of the probability distributions $f(y \mid b)$ (the distribution of $y$ given $b$ ), which is the likelihood considered previously. The distinction is that in $f(y \mid b)$, $y$ is regarded as random for fixed $b$, whereas in $f(b \mid y)$, $b$ is regarded as random for fixed $y$. We saw in the example given in Table 3 that assessments of origin based on $f(y \mid b)$ can lead to incorrect statements concerning $f(b \mid y)$ when the size of the two populations is different.

Bayes Rule relates these two conditional probabilities and clarifies the role of variation in abundance illustrated in Table 3. Bayes Rule states that

$$
f(b \mid y)=\frac{f(y \mid b) f(b)}{f(y)}
$$

where

$$
f(y)=\sum_{b=1}^{B} f(y \mid b) f(b)
$$

which is a constant with respect to $b$ (the random variable in question appearing on the LHS). Therefore, the probability $f(b \mid y)$ is proportional to $f(y \mid b) f(b)$. Whereas $f(y \mid b)$ is the likelihood considered previously, the marginal probability, $f(b)$, describes the (geographic, in the case of isotope markers) distribution of the population being sampled. That is, it describes the probability that a bird chosen at random from the population originates from $b$. It could also be regarded as a prior distribution on origin. For example, if we have no information on $f(y \mid b)$, then the best guess as to the value of $b$, given any particular wintering location, is simply the relative abundance distribution, $f(b)$. For example, if $90 \%$ of a species breeds in region 1 and $10 \%$ in region 2 , and we find one of those birds in Cuba, it seems reasonable to posit, without any additional information whatsoever, that there is a $90 \%$ chance that the bird came from region 1 .

Considering the data in Table $3, f(b)$ is the distribution of abundance among the two populations (rows) so that $f(b=1)=10 / 11$ and $f(b=2)=1 / 11$. Applying Bayes rule yields

$$
\begin{aligned}
f(b & =1 \mid y=3) \\
= & \frac{f(y=3 \mid b=1) f(b=1)}{f(y=3 \mid b=1) f(b=1)+f(y=3 \mid b=2) f(b=2)} \\
= & 0.625 \\
f(b & =2 \mid y=3) \\
& =\frac{f(y=3 \mid b=2) f(b=2)}{f(y=3 \mid b=1) f(b=1)+f(y=3 \mid b=2) f(b=2)} \\
& =0.375 .
\end{aligned}
$$

These posterior probabilities are in agreement with the fact that 30 out of 80 birds (in the population at large) with $y=3$ are from population 2. Conversely, disregarding the information about variable abundance might lead one to (falsely) conclude that $86 \%$ of the $y$ $=3$ birds are from population 2, a marked contrast.

It is clear how this posterior probability of origin relates to the likelihood-based assignment (e.g., Eq. 2). The likelihood assignment implies a uniform prior on $f(b)$. That is, $f(b=1)=f(b=2)=\ldots=f(b=B)$, in which case assignment based on Eq. 2 is equivalent to that based on Eq. 3. Thus, in the probability interpretation given to the likelihood (Eq. 2), we are, in essence, assuming a uniform distribution for $f(b)$, such that the marginal probability of a bird being from any particular breeding origin is the same. This raises an interesting question. Namely, is it sensible to conduct assignment based on a rule that imposes a de facto assumption of constant abundance? In the absence of any information about geographic variation in the breeding population, this is perhaps not unreasonable. However, for many species there may be abundance information available that allows for consideration of other possibilities. In particular, it may be reasonable to use data from existing large-scale monitoring programs to estimate these probabilities, e.g., the North American Breeding Bird Survey, BBS (Robbins et al. 1986). We consider this in the following section.

\section{Abundance AND Black-THRoAted Blue Warblers}

An important difficulty in using abundance information to aid in the assignment of wintering birds is that, for most species, the distribution of the breeding population is unknown. However, information on bird 
TABLE 4. Frequency of origin for birds from four Caribbean islands for posterior assignment (using relative abundance distribution) based on carbon isotopic information alone and using both carbon and hydrogen isotopes.

\begin{tabular}{lcccc}
\hline \hline & \multicolumn{4}{c}{$\begin{array}{c}\text { Wintering capture } \\
\text { location }\end{array}$} \\
\cline { 2 - 5 } $\begin{array}{c}\text { Predicted } \\
\text { origin }\end{array}$ & Cuba & $\begin{array}{c}\text { Dominican } \\
\text { Republic }\end{array}$ & $\begin{array}{c}\text { Puerto } \\
\text { Rico }\end{array}$ & Jamaica \\
\hline$\delta^{13} C$ & & & 37 & 151 \\
$\quad$ Northwest & 49 & 34 & 2 & 38 \\
$\quad$ Northeast & 39 & 4 & 5 & 6 \\
$\quad$ South & 0 & 18 & 16 & 118 \\
$\delta^{13}$ C and $\delta D$ & & & 19 & 48 \\
$\quad$ Northwest & 44 & 23 & 9 & 29 \\
$\quad \begin{array}{l}\text { Northeast } \\
\text { South }\end{array}$ & 43 & 12 & 21 & \\
\hline
\end{tabular}

Note: Data are from Rubenstein et al. (2002).

abundance for most North American species is collected as part of the North American Breeding Bird Survey, (BBS; Robbins et al. 1986). Although imperfect in many respects, the BBS has been used to characterize the breeding distribution of many species. Importantly, for many northern species, the survey does not encompass all of the breeding range. Unfortunately this is the case for the Black-throated Blue Warbler. Although recent BBS coverage has approached the periphery of this species' range, the distribution estimates presented here should be viewed cautiously. Nonetheless, we adopt use of BBS data for characterizing variation in Black-throated Blue Warbler abundance to illustrate the use and effect of abundance information on the assignment to breeding origin.

We created a map of relative abundance of Blackthroated Blue Warblers using a kriging predictor (e.g., Cressie 1991) of the mean BBS route count from 1991 to 2001 , and then computed the distribution of the population among the geographic regions (Fig. 1). In effect, we integrated a map of relative abundance similar to the distribution maps displayed on the U.S. Geological Survey's BBS web site (see the Black-throated Blue Warbler distribution map). ${ }^{4}$ The approximate allocation of Black-throated Blue Warbler abundance among the three regions is 0.500 (Northwest), 0.379 (Northeast), and 0.121 (South). In particular, note that prior odds favor birds originating from "northern", strata ( 1 and 2 ) by about 7 to 1 , or, given a random bird drawn from the population at large, there is roughly an $87 \%$ chance that it originates from the "north."

Using this estimate of $f(b)$ to compute posterior probabilities of origin, and then associating each bird with the region of highest posterior probability, yields the origin distribution given in Table 4 . The origin distribution based on $\delta^{13} C$ alone is substantially different than previously indicated without using abun-

\footnotetext{
4 〈http://www.mbr-pwrc.usgs.gov/bbs/htm96/map617/
} ra6540.html $\rangle$ dance data (i.e., Table 2). In particular, the origin distribution of birds from each island has shifted (in all cases) to favor northern origins, in line with abundance distribution.

Assignment based on both isotopes (Table 4) does reflect more deviation from the prior relative abundance distribution based on just $\delta^{13} C$, and is more consistent with the corresponding results of Table 2 . There is only a slight northern shift compared with the estimated origin distribution that disregards relative abundance information (i.e., Table 2). This is because $\delta D$ is more structured latitudinally, and hence, the assignment outcome is less affected by the prior abundance distribution.

\section{CONClusions AND Discussion}

Stable isotopes have become a common tool for characterizing the movement patterns of migratory birds (reviewed in Rubenstein and Hobson 2004). Isotopic data collected on birds throughout their breeding range can be used to characterize the likelihood of data collected from wintering birds as a function of breeding location. This provides an intuitive means of associating wintering birds to breeding regions (i.e., assignment). We applied this notion to isotopic data from the Black-throated Blue Warbler from Rubenstein et al. (2002) using a likelihood-based assignment approach, and found broad agreement with results reported by them.

Likelihood-based assignment using multiple isotopes should generally yield improved accuracy over a single isotope. In our sample validation exercise, the use of two isotopes did yield improvement in the proportion of individuals correctly assigned to their sample. However, the utility of multiple isotopes depends largely on how geographically structured they are. Our analyses reaffirm the importance of $\delta D$ as a powerful marker for studies of Neotropical migrants. Although much less geographically structured, $\delta^{13} C$ also yielded important information. Data from nitrogen isotopes $\left(\delta^{15} N\right)$ shows no geographic structure in this species (D. Rubenstein, unpublished data), and would not prove useful in improving assignments. However, the incorporation of additional geographically structured isotopes for this species (such as strontium, $\delta^{87} \mathrm{Sr}$; Chamberlain et al. 1997) would improve assignment accuracy. It is important to remember, however, that patterns of isotopic geographic structure will vary over large (i.e., continental) spatial areas and not all isotope markers will work equally well for every species or in each location. For example, although $\delta^{13} C$ has worked well for the Black-throated Blue Warbler, it has not worked well in many other species of Neotropical migrant birds (Hobson et al. 2001, Hobson and Wassenaar 2001, Wassenaar and Hobson 2001). Furthermore, in many Palearctic species that migrate between Eurasia and Africa and molt in Africa, $\delta^{13} C$ and $\delta^{15} N$ show the greatest geographic structure, whereas $\delta D$ shows little 
geographic structure (Chamberlain et al. 2000, Pain et al. 2004, Møller and Hobson, 2004).

Assignment based solely on likelihood ignores information about the distribution of the population, which is informative about origin when abundance is not uniform over the range of the species. That is, lacking any extraneous information about movement patterns, one can reasonably posit that the origin of a bird selected randomly from the population at large (e.g., on its wintering grounds) is in proportion to the geographic distribution of the breeding population. A natural way to accommodate information on the distribution of the population is to consider assignment based on the posterior probability of origin (i.e., Bayes Rule). This is appealing because the assignment problem is more naturally formulated in terms of the probability of origin conditioned on the data (value of a marker) obtained from wintering birds. Then, the probability of origin, given a particular isotope signature, is the product of the likelihood and a prior distribution of origin. This prior distribution is, in effect, the distribution of abundance across the species' range. Failure to consider variation in abundance is a de facto assumption of equal abundance, which may not be desirable (and may even be untenable) for many avian species.

We applied this notion of posterior assignment to the Black-throated Blue Warbler data of Rubenstein et al. (2002) using relative abundance information obtained from the North American Breeding Bird Survey (Robbins et al. 1986). This illustrated one important effect of assignment based on posterior probability: regions of high abundance are favored a priori over regions of low abundance, as they should be. Consequently, the extent to which the resulting (posterior) distribution of origin deviates from the prior is related largely to the magnitude of variation in the isotopic structure among (geographic) populations. As variance among populations increases (i.e., the isotope distributions become more distinguishable), the contribution of relative abundance diminishes. For the Black-throated Blue Warbler data, we note that when using relatively geographically unstructured carbon isotopes, posterior assignment is greatly affected by the distribution of abundance. However, when the more geographically structured hydrogen isotopes are considered, the origin distribution is less sensitive to geographic variation in abundance. These results suggest that relative abundance is likely to be a more crucial consideration in the presence of less geographically structured isotopes, when the distribution of abundance is highly non-uniform, or when the range of the species is geographically restricted. In the latter case, even isotopes with considerable geographic structure at large scales may exhibit relatively low variability at small scales. This will become particularly relevant as stable isotope markers are used to study small-scale animal movements, such as altitudinal migrations and dispersal (Graves et al. 2002, Hobson et al. 2003).

The definition of discrete strata used in our examples is something of an abstraction necessitated by the sparse spatial sampling of isotope ratios in Blackthroated Blue Warbler feathers from breeding sites. Also, some conceptual simplification arises when the problem is developed in terms of discrete populations. One limitation of the discrete model is the improbability of assigning birds to small sources because highabundance sources are favored a priori. Thus, the error rate can be reduced merely be defining a small number of (large) source populations. Conversely, we expect a higher error rate associated with small source populations. The extent to which the degree of aggregation contributes to the outcome depends largely on the magnitude of variation in isotope ratios among source populations. Consider that the odds of correcting associating an observation, $y^{*}$ with the correct source population, $b^{*}$, is

$$
\Omega=\frac{f\left(y^{*} \mid b=b^{*}\right) f\left(b=b^{*}\right)}{f\left(y^{*} \mid b \neq b^{*}\right) f\left(b \neq b^{*}\right)}
$$

which is the product of the likelihood ratio and the prior odds in favor of $b^{*}$. Thus, although the odds in favor of $b^{*}$ decrease with the relative size of the source population, this is moderated by the relative probability of observing $y^{*}$, as it should be (see the example in Table 3). A bird in the population at large does have a small probability of originating from any particular, small, source population; thus, inferences about origin should reflect this unless information is available to suggest otherwise, i.e., unless the likelihood ratio is large relative to the prior odds.

In general, fine-scale inferences about origin will be limited by the information available in isotopic data because of both the variance associated with isotope values at a single location (e.g., Graves et al. 2002) and the fact that there are large geographic areas with similar isotopic baselines (e.g., latitudinal bands of $\delta D)$. However, the advantage of using the posterior assignment framework over existing isotope assignment techniques (e.g., Hobson and Wassenaar 2001, Meehan et al. 2001, Kelly et al. 2002, Rubenstein et al. 2002) is that it facilitates a general probabilistic description of origin. That is, it is an unnecessary simplification to view assignment in terms of discrete source populations; a more general formulation of the problem is possible that renders the aforementioned scale issues irrelevant. For example, finer scale measurements of covariates are available in some applications. These could include factors such as altitude and other landscape or geophysical variables that describe and affect isotope gradients or contours. For isotopes such as $\delta D$, where the isotope ratios in feathers (i.e., feather $\delta D$ ) accurately reflect those in precipitation (i.e., precipitation $\delta D$ ) with a fixed discrimination 
factor of $\sim 25 \%$ (Hobson and Wassenaar 1997, Wassenaar and Hobson 2000, Meehan et al. 2001), a more realistic approach than measuring feather $\delta D$ values at a limited number of sites might be to use existing $\delta D$ base maps of weighted-average growing-season deuterium values for precipitation that reflect samples from a large number of sites (e.g., Hobson and Wassenaar 1997). As other factors such as altitude and local or regional departures from long-term feather $\delta D$ contour base maps are considered (Rubenstein and Hobson 2004), such base maps could be used to estimate origins at increasingly finer scales.

The formulation of a more general assignment framework to accommodate the use of isotope base maps or other continuous-space representations of data is straightforward in principle. To illustrate, suppose that $x(b)$ is a covariate (e.g., $\delta D$ in rainfall or some other geophysical covariate) measured at location $b$ (say, a point on a map). Then we might posit a model for $y(b)$ (isotope ratio in bird feathers) that has mean $\alpha_{0}+$ $\alpha_{1} x(b)$; thus yielding a likelihood $f(y \mid b)$ in terms of the continuous index $b$. Conceptually, $f(y \mid b)$ is simply a regression model relating isotope data in feathers to available covariates (e.g., Hobson and Wassenaar 1997), essentially calibrating isotopes in bird feathers to those in the environment. It should be noted, however, that this will only work for those isotopes that accurately reflect dietary isotope values in animal tissues, and it may be problematic for isotopes where patterns vary with diet, elemental composition, or age (reviewed in Rubenstein and Hobson 2004). Now, given a prior distribution for abundance (a "map"), say $f(b)$, a posterior assignment rule can be obtained by application of Bayes rule as before, yielding a map depicting the probability of origin as a function of breeding location. This does introduce some additional complexity because a general characterization of $f(y \mid b)$ requires that $x(b)$ be observed everywhere within the range of the species. In practice, this may require use of a secondary model for predicting (i.e., mapping) $x(b)$ at unsampled locations. In this context, spatial replication is an important consideration and one must obtain many spatial samples to yield an adequate fit of $f(y \mid b)$ (i.e., estimates of the parameters $\alpha_{0}$ and $\alpha_{1}$ ). Alternatively, this calibration could be addressed independently, based on the geochemistry of the isotopic system, without having to resort to extensive sampling for every species. Thus, using isotope base maps in a probability-based framework for assignment may prove highly useful for studies of avian migration. Specific attention should be paid to precipitation base maps, because feather $\delta D$ values accurately reflect those in precipitation (Hobson and Wassenaar 1997) because $\delta D$ precipitation values are routinely and extensively measured throughout the world (e.g., IAEA/WMO 2001 ), and because $\delta D$ seems to be one of the most informative isotopes for avian movement studies (Rubenstein and Hobson 2004).
As the use of stable isotope markers becomes more widespread in studies of animal movement patterns, it will be essential to consider the geographic structure of isotope signatures in tissues, as well as the number and type of isotopic systems needed. Moreover, we have shown that accounting for relative abundance, which is difficult to estimate for many migratory species, can be important, particularly when few isotopes or those with little geographic structure are used. These new statistical approaches and considerations for analyzing stable isotope data highlight the need for thoughtful and effective experimental design when using isotope markers to study migratory connectivity.

\section{ACKNOWLEDGMENTS}

We thank the participants of the Connectivity of Migratory Birds workshop (held at the Smithsonian Environmental Research Center, Maryland, 2000 and funded by the National Science Foundation) for stimulating many of these ideas. We acknowledge R. Holmes for comments that helped to improve previous versions of this manuscript. We are grateful to $\mathrm{J}$. Pastor, R. Green and an anonymous referee for their constructive reviews of this manuscript. We also thank M. Ayres, C. P. Chamberlain, G. Graves, R. Holmes, N. Tuross, and J. Waldbauer for helping to collect the initial Black-throated Blue Warbler isotope data set. That work was funded by grants from Dartmouth College, the Howard Hughes Medical Institute, and the Andrew W. Mellon Foundation to D. Rubenstein, grants from the National Science Foundation to C. P. Chamberlain and R. Holmes, and from the Smithsonian Institution to G. Graves. D. Rubenstein acknowledges the Howard Hughes Medical Institute for continued support.

\section{Literature Cited}

Chamberlain, C. P., S. Bensch, X. Feng, S. Akesson, and T. Andersson. 2000. Stable isotopes examined across a migratory divide in Scandinavian willow warblers (Phylloscopus trochilus trochilus and Phylloscopus trochilus acredula) reflect their African winter quarters. Proceedings of the Royal Society of London, Series B 267:43-48.

Chamberlain, C. P., J. D. Blum, R. T. Holmes, X. Feng, T. W. Sherry, and G. R. Graves. 1997. The use of isotope tracers for identifying populations of migratory birds. Oecologia 109:132-141.

Cressie, N. A. C. 1991. Statistics for spatial data. John Wiley, New York, New York, USA.

Graves, G. R., C. S. Romanek, and A. R. Navarro. 2002. Stable isotope signature of philopatry and dispersal in a migratory songbird. Proceedings of the National Academy of Sciences (USA) 99:8096-8100.

Hobson, K. A. 2003. Making migratory connections with stable isotopes. Pages 379-391 in P. Berthold and P. Gwinner, editors. Avian migration. Springer-Verlag, Berlin, Germany.

Hobson, K. A. 2004. Flying fingerprints: making connections with stable isotopes and trace elements. In R. Greenberg and P. Marra, editors. Birds of two worlds: the ecology and evolution of migratory birds. Johns Hopkins University Press, Baltimore, Maryland, USA. In press.

Hobson, K. A., K. P. McFarland, L. I. Wassenaar, C. C. Rimmer, and J. E. Goetz. 2001. Linking breeding and wintering grounds of Bicknell's Thrushes using stable isotope analyses of feathers. Auk 118:16-23.

Hobson, K. A., and L. I. Wassenaar. 1997. Linking breeding and wintering grounds of neotropical migrant songbirds using stable hydrogen isotopic analysis of feathers. Oecologia 109:142-148. 
Hobson, K. A., and L. I. Wassenaar. 2001. Isotopic delineation of North American migratory wildlife populations: loggerhead shrikes. Ecological Applications 11:15451553.

Hobson, K. A., L. I. Wassenaar, B. Mila, I. Lovette, C. Dingle, and T. Smith. 2003. Stable isotopes as indicators of altitudinal distributions and movements in an Ecuadorian hummingbird community. Oecologia 136:302-308.

IAEA/WMO (International Atomic Energy Agency/World Meteorological Organization). 2001. Global network for isotopes in precipitation. The GNIP [Global Network of Isotopes in Precipitation] database. 〈http://isohis.iaea.org〉

Kelly, J. F., V. Atudorei, Z. D. Sharp, and D. M. Finch. 2002. Insights into Wilson's Warbler migration from analyses of hydrogen stable-isotope ratios. Oecologia 130:216-221.

Meehan, T. D., C. A. Lott, Z. D. Sharp, R. B. Smith, R. N. Rosenfield, A. C. Stewart, and R. K. Murphy. 2001. Using hydrogen isotope geochemistry to estimate the natal latitudes of immature Cooper's Hawks migrating through the Florida Keys. Condor 103:11-20.

Møller, A. P., and K. A. Hobson. 2004. Heterogeneity in stable isotope profiles predicts coexistence of two populations of barn swallows Hirundo rustica differing in morphology and reproductive performance. Proceedings of the Royal Society of London, Series B, 271:1355-1362.

Nichols, J. D., and R. E. Tomlinson. 1993. Analyses of banding data. Pages 269-280 in T. S. Baskett, M. W. Sayre, R. E. Tomlinson, and R. E. McCabe, editors. Ecology and management of the Mourning Dove. A Wildlife Management Institute Book. Stackpole Books, Harrisburg, Pennsylvania, USA.
Pain, D. J., R. E. Green, B. Gießing, A. Kozulin, A. Poluda, U. Ottosson, M. Flade, and G. M. Hilton. 2004. Using stable isotopes to investigate migratory connectivity of the globally threatened aquatic warbler Acrocephalus paludicola. 138:168-174.

Peterson, B. J. and B. Fry. 1987. Stable isotopes in ecosystem studies. Annual Review of Ecology and Systematics 18: 293-320.

Robbins, C. S., D. A. Bystrak, and P. H. Geissler. 1986. The Breeding Bird Survey: its first fifteen years, 1965-1979. U.S. Department of Interior, Fish and Wildlife Service Resource Publication 157, Washington, D.C., USA.

Rubenstein, D. R., C. P. Chamberlain, R. T. Holmes, M. P. Ayers, J. R. Waldbauer, G. R. Graves, and N. C. Tuross. 2002. Linking breeding and wintering ranges of a migratory songbird using stable isotopes. Science 29:1062-1065.

Rubenstein, D. R., and K. A. Hobson. 2004. From birds to butterflies: animal movement patterns and stable isotopes. Trends in Ecology and Evolution 19:256-263.

Utter, F., and N. Ryman. 1993. Genetic markers and mixed stock fisheries. Fisheries 18:11-21.

Wassenaar, L. I., and K. A. Hobson. 2000. Stable carbon and hydrogen isotope ratios reveal breeding origins of Redwinged Blackbirds. Ecological Applications 10:911-916.

Wassenaar, L. I., and K. A. Hobson. 2001. A stable-isotope approach to delineate geographical catchment areas of avian migration monitoring stations in North America. Environmental Science and Technology 35:1845-1850.

Webster, M. S., P. P. Marra, S. M. Haig, S. Bensch, and R. T. Holmes. 2002. Links between worlds: unraveling migratory connectivity. Trends in Ecology and Evolution 17: 76-83. 